\title{
Synthesis and photo-physical properties of fluorescent 1,3,5-triazine styryl derivatives
}

\author{
Vikas S Padalkar, Vikas S Patil and N Sekar
}

\begin{abstract}

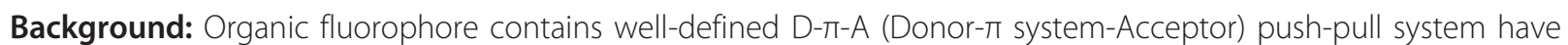
wide application in the field of NLO, OLED and high tech application. Electron donor diphenyl, triphenyl and carbazole conjugated with electron acceptor terminal through $\pi$-system were reported recently for high-tech

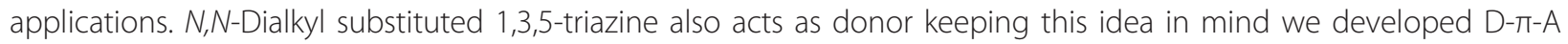
styryl dyes.

Results: Novel "Y"-shaped acceptor- $\pi$-donor- $\pi$-acceptor type of compounds were synthesized from 4,4'-((6-(4(diethylamino)phenyl)-1,3,5-triazine-2,4diyl)bis(oxy)) dibenzaldehyde (DIPOD) as electron donors and different active methylene compounds as electron acceptors by conventional Knoevenagel condensation reaction. Their photophysical and thermal properties were investigated.

Conclusion: It was found that the strong electron acceptor-donor chromophoric system of these compounds showed high Stoke's shift and excellent thermal stability. Compounds showed positive solvatofluorism behavior from nonpolar to polar solvent. All compounds have good thermal stability.
\end{abstract}

\section{Background}

Organic fluorescent heterocyclic chromophores have a wide range of applications in molecular probes [1], fluorescent markers [2], organic light-emitting diodes (OLED)[3], photovoltaic cells [4] and in traditional textile and polymer fields [5]. Electron donors like triphenylamine [6], diphenylamine [7] 1,3,5-triazines [8] and carbazoles [9] with high electron mobility, thermal and photochemical stability are commonly used as holetransporting materials or light-emitting materials for balanced charge injection for above mentioned application.

$N, N$-Dialkyl substituted 1,3,5-triazine fluorescent styryl derivatives have not been exploited much in the past, but attract increasing interest as they exhibit better solubility in common organic solvents, excellent thermal and electron donating ability of $N, N$-alkyl groups. The presence of electron donating $\mathrm{N}, \mathrm{N}$-dialkyl group increases the electron flow towards the electron acceptor moieties present in the molecules. From the viewpoint of

\footnotetext{
* Correspondence: n.sekar@ictmumbai.edu.in Department of Intermediate and Dyestuff Technology, Institute of Chemical Technology (Formerly UDCT), N. P. Marg, Matunga, Mumbai-400 019, Maharashtra, India
}

molecular design, structural features such as the long $\pi$ conjugation style, molecular planarity and length of the conjugated bridge play important roles in functional chromophores, which are highly promising for electronic applications like photonic materials $[10,11]$, holographic optical data storage [12] and organic photorefractive materials.

4-(4,6-Dichloro-1,3,5-triazin-2-yl)- $N, N$-diethylaniline is structurally similar to 4,4'-disubstituted diphenylamine and 3,6-disubstituted carbazoles with planar structure. The 4-(4,6-dichloro-1,3,5-triazin-2-yl)- N,N-diethylaniline nucleus can be easily functionalized at 4,6- positions and covalently linked to other molecular groups. A typical push-pull chromophore consists of a polar A- $\pi-D$ system with a planar $\pi$-system end-capped by a strong electron donor (D) and a strong electron acceptor (A). The $\pi$-conjugated system ensuring intramolecular charge transfer (ICT) between the donor ( $\mathrm{D}=\mathrm{N}, \mathrm{N}$-dialkyl group) and the acceptor $(\mathrm{A}=-\mathrm{CN}$, COOEt group etc.) is the most common of conjugated double or triple bonds in aromatic and heteroaromatic rings as well as their combinations $[13,14]$.

There has always been an effort to design and synthesize novel and well-defined organic push-pull 
system with prospective applications as chromophores for nonlinear optics (NLO), electronic and photonic devices, organic light-emitting diodes (OLED) and functional polymers [15]. In this paper, synthesis of fluorescent styryl dyes, their structure elucidation and the photophysical and thermal properties are reported.

\section{Results and Discussion}

\section{Synthesis and Characterization of compounds}

The six novel fluorescent triazine based styryl compounds were prepared Figure 1 by classical Knoevenagel condensation of 4,4'-((6-(4-(diethylamino) phenyl)1,3,5-triazine-2,4 diyl) bis (oxy)) dibenzaldehyde 5 with active methylene compounds $\mathbf{6 a - 6} \mathbf{f}$ as shown in Scheme 1. In the first step, the 4-(4,6-dichloro-1,3,5triazin-2-yl)-N,N-diethylaniline (3) was synthesized from cyanuric chloride $\mathbf{1}$ and $\mathrm{N}, \mathrm{N}$-diethyl aniline $\mathbf{2}$. Finally aldehyde $\mathbf{5}$ and suitable active methylene compound 6a-6f were stirred in absolute ethanol containing a catalytic amount of piperidine to yield desired styryl triazines $7 \mathbf{a}-\mathbf{7 f}$. The structures of the compounds were confirmed by FT-IR, ${ }^{1} \mathrm{H}$ NMR, ${ }^{13} \mathrm{C}$ NMR, and Mass spectral analysis. The ${ }^{1} \mathrm{H}$ NMR spectra of compounds 5 showed singlet peak at $\delta 10.04$ which indicates the confirmation of compounds 5 and singlet $\delta$ 7.76 to 8.46 in $7 \mathbf{a}-7 \mathbf{f}$ which indicates the ethylenic protons at styryl group.

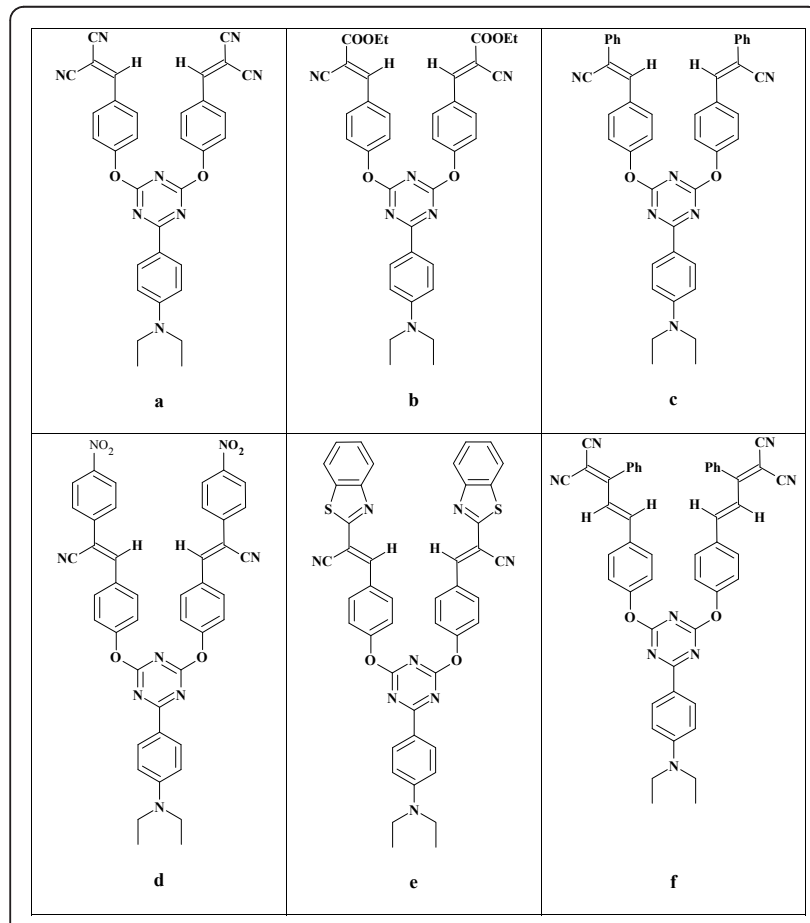

Figure 1 Synthesized styryl derivatives $7 a-7 f$

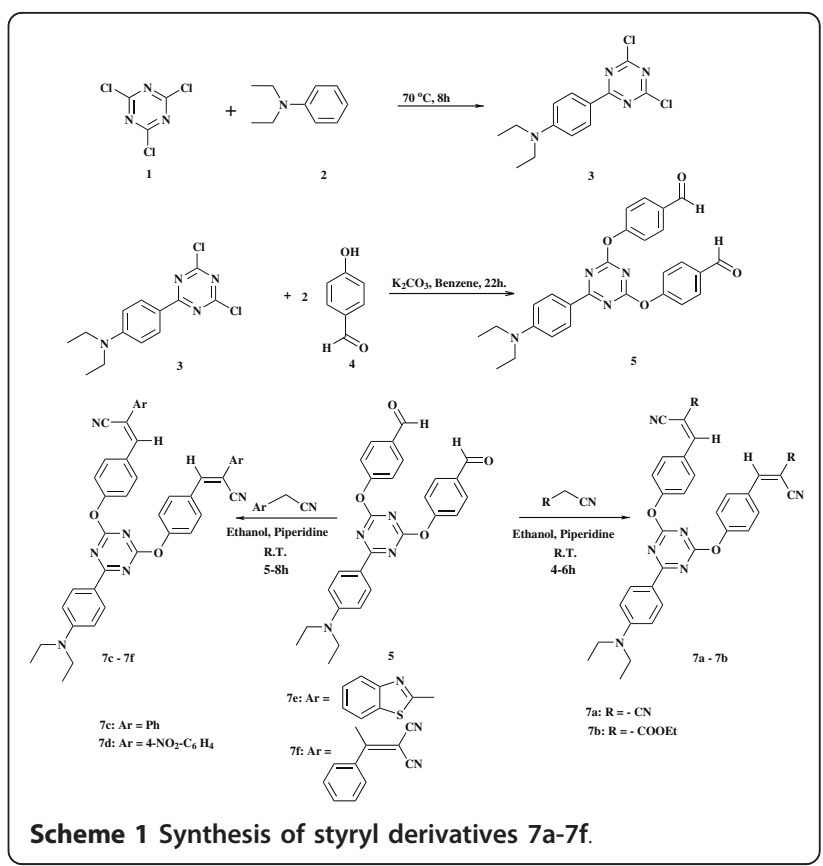

\section{Photo-physical properties}

The UV-Vis absorption and emission spectra of styryl derivatives $(\mathbf{7 a - 7} \mathbf{f})$ were recorded in acetonitrile at room temperature and the compound concentration is $1 \times 10^{-}$ ${ }^{6} \mathrm{M}$. The $\lambda \max$ (absorbance) values of compounds (7a$7 \mathbf{f})$ were obtained as $(7 \mathbf{a}=381 ; 7 \mathbf{b}=378 ; 7 \mathbf{c}=354 ; 7 \mathbf{d}$ $=354 ; 7 \mathbf{e}=354 ; 7 \mathbf{f}=369) \mathrm{nm}$ Figure 2 and fluorescence emission of compounds $7 \mathbf{a}-7 \mathbf{f}$ were obtained as 422, 413, 408, 413, 399 and $403 \mathrm{~nm}$ respectively with difference in fluorescence intensity Figure 3.

To evaluate the effect of solvent polarity on absorptionemission properties of synthesized compounds. Absorption, emission properties of compound $7 \mathbf{a}-7 \mathbf{f}$ were studied in different solvents of varying polarity and

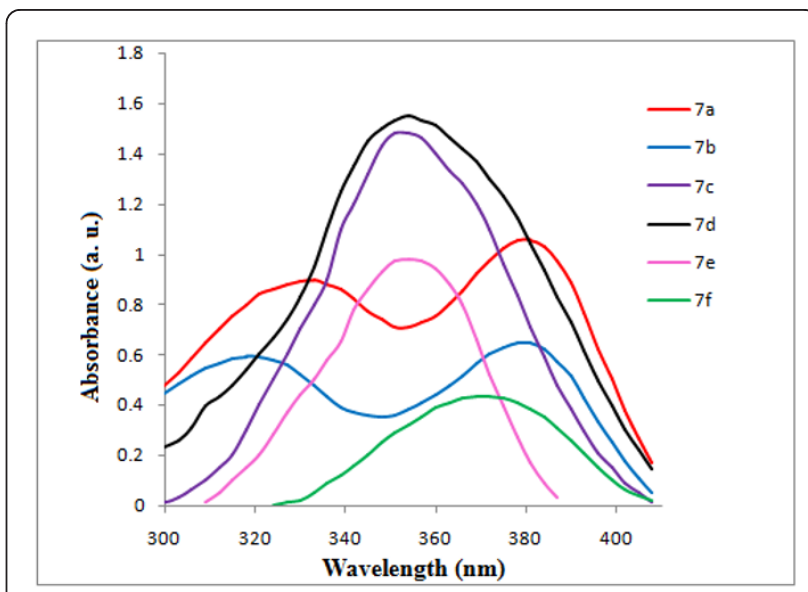

Figure 2 UV-Visible absorption spectra of compounds 7a-7f 


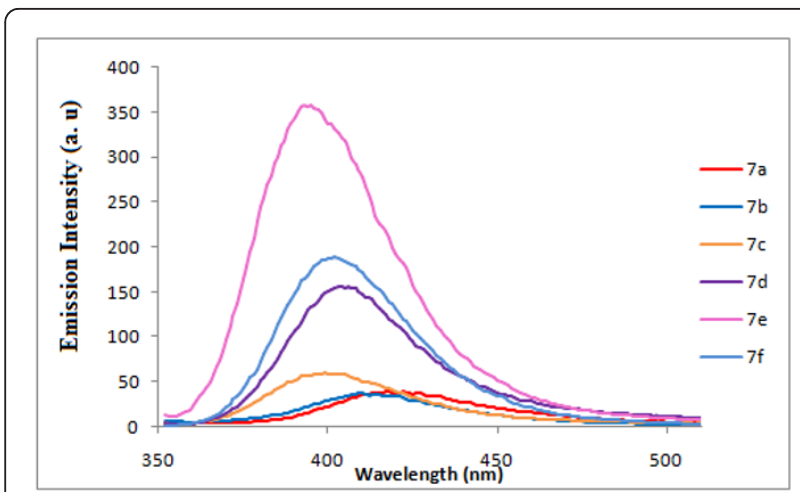

Figure 3 Fluorescence emission spectra of compounds 7a-7f. hydrogen bonding capability as shown in Table 1 . Six solvents are tested for effect of solvent on their absorptionemission characteristics. The absorption-emission spectrums of $7 \mathbf{a}-\mathbf{7 f}$ are not affected much by a change in polarity and hydrogen bonding capacity of the solvent.

These compounds with A- $\pi-\mathrm{D}$ structure consist of an electron-donating $\mathrm{N}, \mathrm{N}$-diethyl group on triazine moiety and electron withdrawing cyano or carboethoxy group. The results showed that these compounds do not show strong solvatochromic and solvatofluoric properties. The introduction of electron accepting groups in the triazine moiety causes intramolecular charge transfer and mesomeric dipole moment. Depending upon the electron affinity of acceptor groups, the CT band was slightly shifted
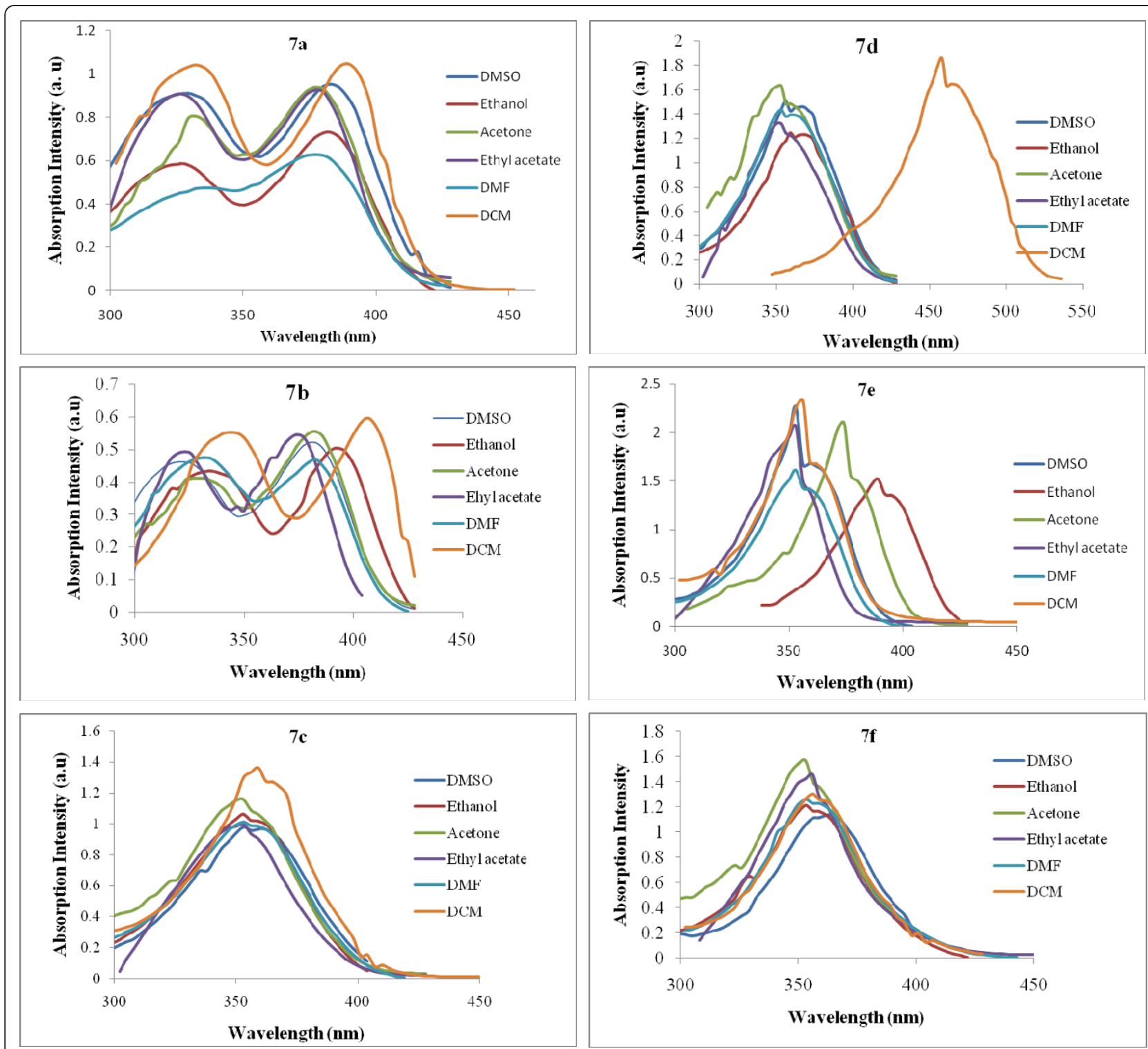

Figure 4 Effect of solvent polarity on absorption spectra of compounds 7a-7f. 
Table 1 Fluorescence absorption and emission maxima of compound $7 \mathrm{a}-7 \mathrm{f}$ in various solvents.

\begin{tabular}{|c|c|c|c|c|c|c|c|c|c|}
\hline \multirow[t]{2}{*}{ Solvents } & \multicolumn{3}{|c|}{$7 a$} & \multicolumn{3}{|c|}{$7 b$} & \multicolumn{3}{|c|}{$7 c$} \\
\hline & $\lambda \max$ & $\lambda$ em & $\Delta \lambda$ & $\lambda \max$ & $\lambda$ em & $\Delta \lambda$ & $\lambda \max$ & $\lambda$ em & $\Delta \lambda$ \\
\hline $\mathrm{DCM}$ & $\begin{array}{l}329(1.030) \\
389(1.048)\end{array}$ & 408(079) & 79 & $\begin{array}{l}341(0.549) \\
404(0.589)\end{array}$ & 403(149) & 62 & $359(1.361)$ & 408(482) & 49 \\
\hline EtOAc & $\begin{array}{l}323(0.898) \\
377(0.852)\end{array}$ & 403(490) & 80 & $\begin{array}{l}320(0.488) \\
371(0.531)\end{array}$ & 392(201) & 87 & $356(0.956)$ & 403(490) & 47 \\
\hline Acetone & $\begin{array}{l}329(0.793) \\
377(0.927)\end{array}$ & $406(688)$ & 77 & $\begin{array}{l}323(0.375) \\
380(0.550)\end{array}$ & $410(108)$ & 72 & $354(1.157)$ & 403(216) & 49 \\
\hline EtOH & $\begin{array}{l}326(0.585) \\
380(0.727) \\
\end{array}$ & $428(288)$ & 102 & $\begin{array}{l}332(0.420) \\
389(0.503) \\
\end{array}$ & $424(131)$ & 92 & $353(1.061)$ & $410(166)$ & 57 \\
\hline DMF & $\begin{array}{l}329(0.458) \\
377(0.628)\end{array}$ & $401(487)$ & 72 & $\begin{array}{l}332(0.475) \\
380(0.461)\end{array}$ & 392(183) & 60 & $350(0.994)$ & $411(132)$ & 61 \\
\hline DMSO & $\begin{array}{l}326(0.908) \\
383(0.982)\end{array}$ & $428(087)$ & 102 & $\begin{array}{l}323(0.455) \\
377(0.539)\end{array}$ & $418(229)$ & 95 & $353(0.979)$ & $418(164)$ & 65 \\
\hline \multirow[t]{2}{*}{ Solvents } & \multicolumn{3}{|c|}{$7 d$} & \multicolumn{3}{|c|}{$7 e$} & \multicolumn{3}{|c|}{$7 f$} \\
\hline & $\lambda \max$ & $\lambda$ em & $\Delta \lambda$ & $\lambda \max$ & $\lambda$ em & $\Delta \lambda$ & $\lambda \max$ & $\lambda$ em & $\Delta \lambda$ \\
\hline DCM & $410(1.864)$ & $458(1000)$ & 48 & $356(2.322)$ & $400(1000)$ & 44 & $356(1.305)$ & $416(1000)$ & 60 \\
\hline EtOAc & 353(1.319) & $410(1000)$ & 57 & $350(1.992)$ & $410(1000)$ & 60 & $353(1.424)$ & $416(1000)$ & 63 \\
\hline Acetone & $350(1.621)$ & $410(1000)$ & 60 & $374(2.099)$ & $400(1000)$ & 26 & $353(1.571)$ & 408(684) & 55 \\
\hline EtOH & $362(1.209)$ & $418(600)$ & 56 & $389(1.521)$ & $408(1000)$ & 19 & $353(1.213)$ & 413(937) & 57 \\
\hline DMF & $356(1.380)$ & $413(561)$ & 57 & $353(1.616)$ & $403(681)$ & 50 & $353(1.229)$ & $413(363)$ & 60 \\
\hline DMSO & $356(1.507)$ & $420(400)$ & 64 & $353(2.252)$ & $408(476)$ & 55 & $359(1.166)$ & $416(359)$ & 57 \\
\hline
\end{tabular}

DCM: Dichloromethane; EtOAc: Ethyl acetate; EtOH: Ethyl alcohol; DMF: Dimethyforamide; DMSO: Dimethylsulphoxide.

in DMF with decrease Stoke's shift as shown in Table 1. The absorption and emission spectra of dye $7 \mathbf{a}-7 \mathbf{f}$ in several solvents having different polarities are shown in Figures 4 and 5 . Compound $7 \mathbf{a}$ and $7 \mathbf{b}$ shows dual absorption in dichloromethane and ethanol, while compound $\mathbf{7 d}$ shows red shift in dichloromethane and $7 \mathbf{e}$ shows red shift in acetone and ethanol. The negative solvatochromism observed in $\mathbf{7} \mathbf{d}$ indicates that there is no charge transfer character in the molecule after excitation; therefore presence of nitro group does not lower the energy of the excited state by solvation. In the case of 7e presence of benzothiazolyl ring gives a charge transfer characteristic to the system and thus exhibits positive solvatochromism.

An effective fluorescent dyes for biological application should have good fluorescence intensity, high quantum yield and high photostability. Quantum yield of compounds $7 \mathbf{a}-7 \mathbf{f}$ were determined by using anthracene as standard. Absorption and emission characteristics of standard as well as unknown samples were measured at different concentration of unknown samples and standard at $(2,4,6,8$ and $10 \mathrm{ppm}$ level). Graphs were plotted absorbance intensity values against emission intensity values. A linear plot was obtained. Gradients were calculated for each unknown compound and for standards. All the measurements were done by keeping the parameters constant such as solvent and slit width. Relative quantum yield of all synthesized compounds $7 \mathbf{a}-7 \mathbf{f}$ were calculated by using Formula 1[16].

Formula 1: Relative fluorescence quantum yield

$$
\Phi_{\mathrm{X}}=\Phi_{\mathrm{ST}}\left(\operatorname{Grad}_{\mathrm{x}} / \operatorname{Grad}_{\mathrm{ST}}\right)\left(\eta^{2} \mathrm{x} / \eta_{\mathrm{ST}}^{2}\right)
$$

Where: $\Phi_{\mathrm{X}}=$ Quantum yield of unknown sample

$\Phi_{\mathrm{ST}}=$ Quantum yield of standard used

$\operatorname{Grad}_{\mathrm{X}}=$ Gradient of unknown sample

$\operatorname{Grad}_{\mathrm{X}}=$ Gradient of standard used

$\eta_{\text {ST }}^{2}=$ Refractive index of solvent for standard sample

$\eta^{2} x=$ Refractive index of solvent for sample

The fluorescence quantum yields of $7 \mathbf{a}-7 \mathbf{f}$ were recorded in ethanol at room temperature. It was observed that the quantum yield of $7 \mathbf{d}$ and $7 \mathbf{e}$ in ethanol (0.0039 for $7 \mathbf{a}, 0.0026$ for $7 \mathbf{b}, 0.0082$ for $7 \mathbf{c}, 0.0689$ for $7 \mathbf{d}, 0.0291$ for $7 \mathbf{e}$ and 0.0034 for $7 \mathbf{f}$ ) are higher than $7 \mathbf{a}-$ $7 \mathbf{c}$ and $7 \mathbf{f}$ in the same solvent. The details are given in the Table 2. Quantum yield results summarized in Table 2 indicate that, compounds $7 \mathbf{d}$ and $7 \mathbf{e}$ show high quantum efficiency as compared to compound $7 \mathbf{a}, \mathbf{7 b}$, $\mathbf{7 c}$ and $\mathbf{7 f}$. This difference in quantum efficiency is may be due to conjugation through aromatic system as well as strong hydrogen bonding between nitro of $\mathbf{7} \mathbf{d}$ and ethanol, and thiazole of $7 \mathbf{e}$ and ethanol.

\section{Thermal Properties}

In order to examine the thermal stability of these compounds $7 \mathbf{a}-7 \mathbf{f}$ thermo gravimetric (TG) and differential 

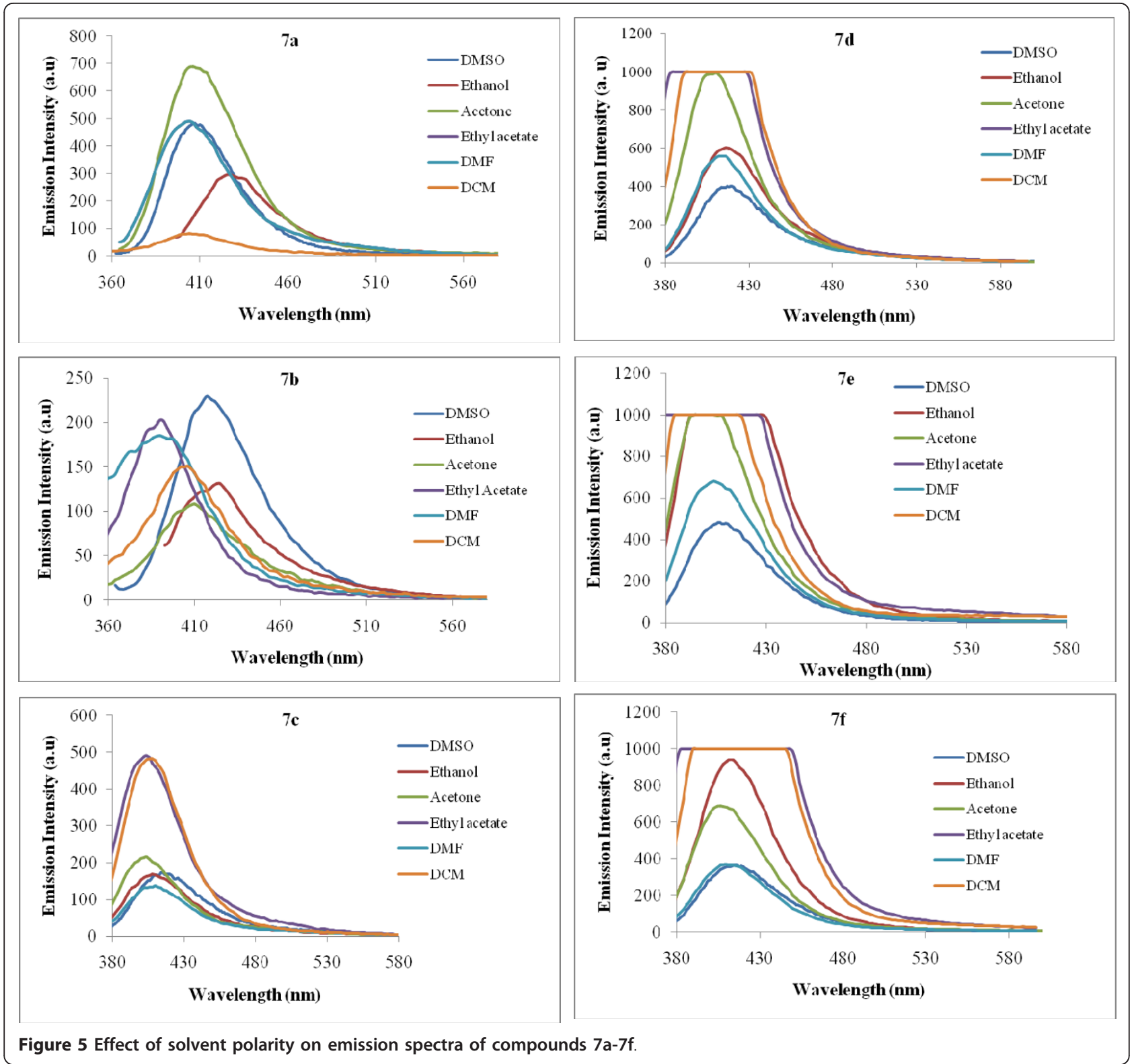

scanning colorimeter (DSC) analysis were carried out between $40-600^{\circ} \mathrm{C}$ under a nitrogen atmosphere. The TG curves of the compounds are shown in Figure 6. The TG results indicates that the frame work of the synthesized compounds are stable up to $300^{\circ} \mathrm{C}$. Above $250^{\circ} \mathrm{C}$ the thermo gravimetric curves of the synthesized compounds show a loss in weight. The comparisons of the $T_{d}$ (decomposition temperature) showed that the thermal stability of $\mathbf{7 a}-7 \mathbf{f}$ decreases in the order $7 \mathbf{c}>7 \mathbf{a}>\mathbf{7 f}>\mathbf{7 b}>\mathbf{7 d}>\mathbf{7 e}$. Thermal stability graph as shown in Figure 6. Compound $7 \mathbf{a}$ is thermally more stable, it does not decompose completely upto $600^{\circ} \mathrm{C}\left(65 \%\right.$ stable up to temperature $\left.600^{\circ} \mathrm{C}\right)$. Compounds $7 \mathbf{a}$ and $7 \mathbf{b}$ are $40 \%$ stable up to temperature $600^{\circ} \mathrm{C}$. Compounds $7 \mathbf{c}, 7 \mathbf{e}$ and $7 \mathbf{f}$ decompose almost
Table 2 Absorption, emission and quantum yield of compounds 7a-7f.

\begin{tabular}{ccccc}
\hline Compounds & $\begin{array}{c}\text { Absorption }^{\text {a }} \\
\text { Intensity } \\
(\mathbf{a} . \mathbf{u} \text {.) } \\
\lambda \max (\mathbf{n m})\end{array}$ & $\begin{array}{c}\text { Emission }^{\text {a }} \\
\text { Intensity } \\
(\mathbf{a} . \mathbf{u} \text {.) } \\
\boldsymbol{\lambda m a x ( n m )}\end{array}$ & $\begin{array}{c}\text { Stoke } \\
\text { Shift } \\
(\boldsymbol{\Delta} \boldsymbol{\lambda})\end{array}$ & $\begin{array}{c}\text { Quantum } \\
\text { Yield }^{\mathbf{b}}\end{array}$ \\
\hline 7a & $381(1.056)$ & $422(038)$ & 41 & 0.0039 \\
7b & $378(0.645)$ & $413(036)$ & 35 & 0.0026 \\
7c & $354(1.509)$ & $408(057)$ & 54 & 0.0082 \\
7d & $354(1.547)$ & $413(149)$ & 59 & 0.0689 \\
7e & $354(1.025)$ & $399(344)$ & 45 & 0.0295 \\
7f & $369(0.434)$ & $403(182)$ & 34 & 0.0034 \\
\hline
\end{tabular}

${ }^{a}$ Absorption and Emission were measured in acetonitrile.

${ }^{\mathrm{b}}$ Solvent for quantum yield measurement in ethanol. 


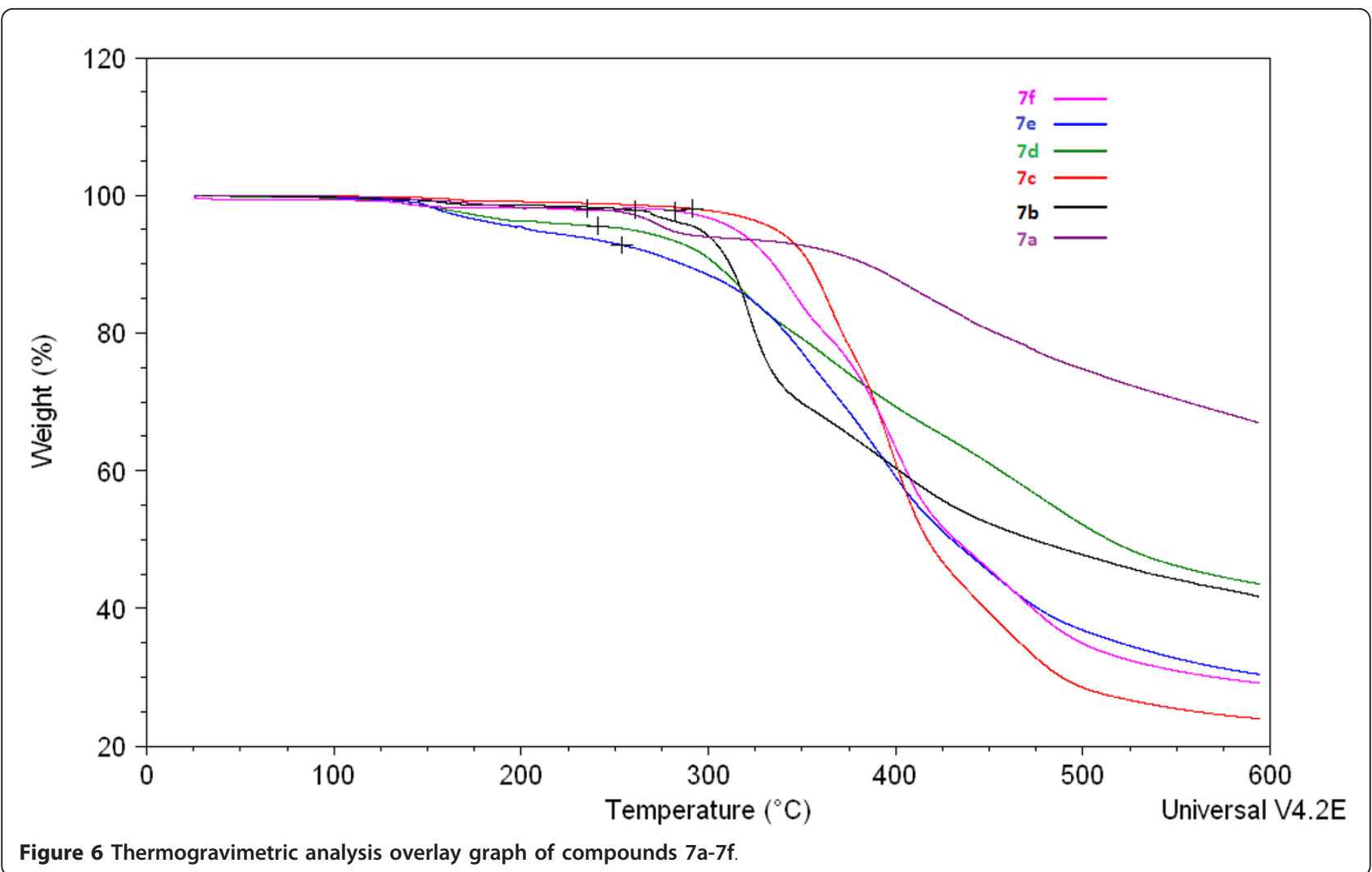

completely beyond temperature $600^{\circ} \mathrm{C} .7 \mathrm{~d}$ and $7 \mathrm{e}$ starts decomposing at $120^{\circ} \mathrm{C}$ but after $300^{\circ} \mathrm{C}$ its decomposition behavior is same like $7 \mathbf{a}, 7 \mathbf{b}, 7 \mathbf{c}$ and $\mathbf{7 f}$.

\section{Experimental}

\section{Materials and equipments}

All commercial reagents and solvents were procured from s. d. fine chemicals (India) and were used without purification. The reaction was monitored by TLC using on $0.25 \mathrm{~mm}$ E-Merck silica gel $60 \mathrm{~F}_{254}$ precoated plates, which were visualized with UV light. Melting points were measured on standard melting point apparatus from Sunder industrial product Mumbai, and are uncorrected. The FT-IR spectra were recorded on Perkins-Elmer 257 spectrometer using $\mathrm{KBr}$ discs. ${ }^{1} \mathrm{H}$-NMR and ${ }^{13} \mathrm{C}$ NMR spectra were recorded on VARIAN 400$\mathrm{MHz}$ instrument (USA) using TMS as an internal standard. Mass spectra were recorded on Finnigan mass spectrometer. The visible absorption spectra of the compounds were recorded on a Spectronic Genesys 2 UV-Visible spectrometer. Simultaneous DSC-TGA measurements were performed out on SDT Q $600 \mathrm{v} 8.2$ Build 100 model of TA instruments Waters (India) Pvt. Ltd.

\section{Synthesis and characterization}

Synthesis of 4-(4,6-dichloro-1,3,5-triazin-2-yl)N,Ndiethylaniline 3

A mixture of $N, N$-diethylaniline $1(27.0 \mathrm{~g}, 0.2 \mathrm{~mol})$ and cyanuric chloride $2(18.4 \mathrm{~g}, 0.1 \mathrm{~mol})$ was heated at $70^{\circ} \mathrm{C}$ for $8 \mathrm{~h}$ under a slow stream of dry nitrogen. After completion of reaction monitored by TLC, the mixture was extracted with hot chloroform $(200 \mathrm{~mL})$ and the white crystalline hydrochloride salt of $N, N$-diethylaniline removed by filtration. Slow cooling and evaporation of the chloroform extract to a volume of $50 \mathrm{~mL}$ yielded good crystals of 3 . The product was recrystallized two times from acetone.

Yield: 11.68 g, 40\%; m.p.: $156^{\circ} \mathrm{C}$. (Recrystallized from acetone)

FT-IR (KBr) v max: 567, 715, 824, 839, 1164, 1232, 1515, 1610, 2967, $3411 \mathrm{~cm}^{-1}$

${ }^{1}$ H NMR (400 MHz) (ס: ppm): 1.22-1.25 (t, 6H, $\left.-\mathrm{CH}_{3}\right), 3.44-3.46\left(\mathrm{q}, 4 \mathrm{H},-\mathrm{CH}_{2}\right), 6.65-6.69$ (dd, $2 \mathrm{H}, J=$ 9.2, $2.8 \mathrm{~Hz}, \mathrm{Ar}-\mathrm{H}), 8.29-8.33(\mathrm{dd}, 2 \mathrm{H}, J=9.2,2.8 \mathrm{~Hz}$, Ar-H).

${ }^{13}$ C NMR (100 MHz) (ס: ppm): 15.6, 49.0, 114.6, 125.6, 130.7, 154.2, 172.3, 179.5.

Mass: $\mathrm{m} / \mathrm{e}=298(\mathrm{M}+1), 299(\mathrm{M}+2)$ 
4,4'-((6-(4-(N,N-Diethylamino) phenyl)-1,3,5-triazine-2,4 diyl)bis(oxy)) dibenzaldehyde 5 -Hydroxybenzaldehyde $4(2.42 \mathrm{~g}, 0.022 \mathrm{~mol})$ and 4-(4,6dichloro-1,3,5-triazin-2-yl)- $N, N$-diethylaniline 3 (3.0 g, $0.011 \mathrm{~mol}$ ) were added to a suspension of $\mathrm{K}_{2} \mathrm{CO}_{3}(3.04$ g, $0.022 \mathrm{~mol}$ ) in $50 \mathrm{~mL}$ of benzene. The mixture was refluxed for $22 \mathrm{~h}$. The reaction mixture was then cooled and the solid was removed by filtration and washed with hot ethyl acetate twice. The filtrate was extracted with $10 \% \mathrm{Na}_{2} \mathrm{CO}_{3}$ solution $(20 \mathrm{~mL})$ twice and with $\mathrm{H}_{2} \mathrm{O}$ once. The organic layer was dried over anhydrous $\mathrm{Na}_{2} \mathrm{SO}_{4}$ and then concentrated. The white powder was recrystallized from $20 \mathrm{~mL}$ of ethanol to afford $3.31 \mathrm{~g}$ of 4,4'-((6-(4-( $N, N$-diethylamino)phenyl)1,3,5-triazine-2,4 diyl)bis(oxy)) dibenzaldehyde 5 as a white fluffy precipitate $(80 \%)$.

Yield: $3.31 \mathrm{~g}, 80 \%$; m. p.: $150^{\circ} \mathrm{C}$.

FT-IR (KBr) v max.: 505, 726, 831, 1565, 1705(C = O), 2729, 2929(Aldehyde CH), $2972 \mathrm{~cm}^{-1}$.

${ }^{1} \mathrm{H}$ NMR (400 MHz) (ס: ppm): $1.19\left(\mathrm{t}, 6 \mathrm{H},-\mathrm{CH}_{3}\right)$, $3.43\left(\mathrm{q}, 4 \mathrm{H},-\mathrm{CH}_{2}\right), 6.58(\mathrm{~d}, 1 \mathrm{H}, J=9.17,2.71 \mathrm{~Hz}, \mathrm{Ar}-$ $\mathrm{H}), 6.61(\mathrm{~d}, 1 \mathrm{H}, J=9.17,2.71 \mathrm{~Hz}, \mathrm{Ar}-\mathrm{H}), 7.41(\mathrm{~d}, 2 \mathrm{H}, J$ $=8.43,1.83 \mathrm{~Hz}, \mathrm{Ar}-\mathrm{H}), 7.44(\mathrm{~d}, 1 \mathrm{H}, J=8.43,1.83 \mathrm{~Hz}$, $\mathrm{Ar}-\mathrm{H}), 7.95(\mathrm{~d}, 1 \mathrm{H}, J=8.80,1.83 \mathrm{~Hz}, \mathrm{Ar}-\mathrm{H}), 7.98(\mathrm{~d}$, $1 \mathrm{H}, J=8.80,1.83 \mathrm{~Hz}, \mathrm{Ar}-\mathrm{H}), 8.05(\mathrm{~d}, 1 \mathrm{H}, J=9.17 \mathrm{~Hz}$, Ar-H), $8.08(\mathrm{~d}, 1 \mathrm{H}, J=9.17 \mathrm{~Hz}, \mathrm{Ar}-\mathrm{H}), 10.04(\mathrm{~s}, 2 \mathrm{H}$, Aldehyde $\mathrm{H})$.

${ }^{13} \mathrm{C}$ NMR (100 MHz)(ס: ppm):12.37, 43.92, 110.72, 119.13, 122.56, 130.91, 131.13, 133.76, 151.58, 156.25, $171.53,174.40,192.04$.

Mass: $\mathrm{m} / \mathrm{e}=469.2\left(\mathrm{M}^{+}+1\right)$.

Synthesis of Styryl 7a-7f Synthesis of 2,2'-((6-(4- $(N, N-$ diethylamino)phenyl)-1,3,5-triazine-2,4-diyl)bis(oxy)) bis(4,1-phenylene)bis(methanylylidene)dimalononitrile $7 \mathrm{a}$

4,4'-((6-(4-(N,N-Diethylamino) phenyl)-1,3,5-triazine2,4 diyl) bis (oxy)) dibenzaldehyde 5 (1.0 g, $0.0023 \mathrm{~mol})$ and malanonitrile $(0.33 \mathrm{~g}, 0.0052 \mathrm{~mol})$ were dissolved in absolute ethanol $(10 \mathrm{~mL})$. Piperidine $(0.1 \mathrm{~mL})$ was added to it and the reaction mixture was stirred at room temperature for $4 \mathrm{~h}$. filtered the reaction mass to separate out product and recrystallized it from ethanol.

Yield: $(0.96$ g, $78 \%)$, m.p.: $198^{\circ} \mathrm{C}$.

FT-IR (KBr) v max: 805, 1138, 1174, 1233, 1344, 1541, 2229, 2902, $2986 \mathrm{~cm}^{-1}$.

${ }^{1} \mathrm{H}$ NMR (400 MHz) (ס: ppm): $1.23\left(\mathrm{t}, 6 \mathrm{H},-\mathrm{CH}_{3}\right)$, $3.44\left(\mathrm{q}, 4 \mathrm{H},-\mathrm{CH}_{2}\right), 6.60-6.63(\mathrm{~d}, 2 \mathrm{H}, J=9.4 \mathrm{~Hz}, \mathrm{Ar}-\mathrm{H})$, 7.43-7.47 (d, $4 \mathrm{H}, J=1.8,8.7 \mathrm{~Hz}, \operatorname{Ar}-\mathrm{H}), 7.79(\mathrm{~s}, 2 \mathrm{H}$, Ethylenic protons), 8.00-8.03 (d, $2 \mathrm{H}, J=1.8,8.8 \mathrm{~Hz}, \mathrm{Ar}-$ $\mathrm{H}), 8.06-8.07(\mathrm{~d}, 2 \mathrm{H}, J=7.0,2.5 \mathrm{~Hz}, \mathrm{Ar}-\mathrm{H}), 8.10(\mathrm{~d}, 2 \mathrm{H}$, $J=2.2 \mathrm{~Hz}, \mathrm{Ar}-\mathrm{H})$.

${ }^{13}$ C NMR (100 MHz) (ס: ppm):12.38, 43.94, 81.13, $110.72,113.28,114.26,119.17,122.94,128.79,130.98$, $132.28,151.64,155.84,160.40,171.39,174.55$.
Mass: $\mathrm{m} / \mathrm{e}=565.2(\mathrm{M}+1), 566.2(\mathrm{M}+2)$.

2E, 2'E-Diethyl-3,3'-((6-(4-( $N, N$-diethylamino)phenyl)-1,3,5-triazine-2,4-diyl)bis(oxy))bis(4,1-phenylene) bis(2-cyanoacrylate) 7 b.

4,4'-((6-(4-(N,N-Diethylamino) phenyl)-1,3,5-triazine2,4 diyl) bis (oxy)) dibenzaldehyde 5 (1.0 g, $0.0023 \mathrm{~mol})$ and ethylcyanoacetate $(0.59 \mathrm{~g}, 0.0052 \mathrm{~mol})$ were dissolved in absolute ethanol $(10 \mathrm{~mL})$. Piperidine $(0.1 \mathrm{~mL})$ was added to it and the reaction mixture was stirred at room temperature for $5 \mathrm{~h}$ filtered the reaction mass to separate out product and recrystallized product from ethanol.

Yield: (1.05 g, 71\%), Melting Point: $162-164^{\circ} \mathrm{C}$.

FT-IR (KBr) v max.: 512, 729, 831, 1134, 1565, 1630, 1729, 2735, 3075, $3390 \mathrm{~cm}^{-1}$.

${ }^{1} \mathrm{H}$ NMR (400 MHz) (ס: ppm): $1.20\left(\mathrm{t}, 6 \mathrm{H},-\mathrm{CH}_{3}\right)$, $1.42\left(\mathrm{t}, 6 \mathrm{H},-\mathrm{CH}_{3}\right), 3.41\left(\mathrm{q}, 4 \mathrm{H},-\mathrm{CH}_{2}\right), 4.41(\mathrm{q}, 4 \mathrm{H}$, $\left.-\mathrm{CH}_{2}\right), 6.60-6.63(\mathrm{~d}, 2 \mathrm{H}, J=9.52 \mathrm{~Hz}, \mathrm{Ar}-\mathrm{H}), 7.39$ (d, $2 \mathrm{H}, J=8.8 \mathrm{~Hz}, \mathrm{Ar}-\mathrm{H}), 7.42(\mathrm{~d}, 2 \mathrm{H}, J=8.8 \mathrm{~Hz}, \mathrm{Ar}-\mathrm{H})$, $8.08(\mathrm{~d}, 2 \mathrm{H}, J=8.8,4.0 \mathrm{~Hz}, \mathrm{Ar}-\mathrm{H}), 8.09(\mathrm{~d}, 2 \mathrm{H}, J=4.0$ $\mathrm{Hz}, \mathrm{Ar}-\mathrm{H}), 8.10$ (d, $2 \mathrm{H}, J=8.8,4.0 \mathrm{~Hz}, \mathrm{Ar}-\mathrm{H}), 8.26(\mathrm{~s}$, $2 \mathrm{H}$, Ethylenic protons).

${ }^{13}$ C NMR (100 MHz) (ס: ppm): 12.40, 14.01, 43.94, $62.41,102.24,110.74,115.68,122.77,128.89,130.95$, 132.49, 154.06, 155.17, 161.84, 171.51

Mass: $\mathrm{m} / \mathrm{e}=659.3(\mathrm{M}+1), 660.3(\mathrm{M}+2)$.

2E, 2'E-3,3'-((6-(4-( $N, N$-Diethylamino)phenyl)-1,3,5triazine-2,4-diyl)bis(oxy))bis(4,1-phenylene)bis(2-phenylacrylonitrile) $7 \mathrm{c}$.

4,4'-((6-(4-(N,N-Diethylamino) phenyl)-1,3,5-triazine2,4 diyl) bis (oxy)) dibenzaldehyde 5 (1.0 g, $0.0023 \mathrm{~mol})$ and phenyl acetonitrile $(0.61 \mathrm{~g}, 0.0052 \mathrm{~mol})$ were dissolved in absolute ethanol $(10 \mathrm{~mL})$. Piperidine $(0.1 \mathrm{~mL})$ was added to it and the reaction mixture was stirred at room temperature for $6 \mathrm{~h}$ filtered the reaction mass to separate out product and recrystallized product from ethanol.

Yield: $(1.10 \mathrm{~g}, 76 \%)$, m.p.: $160^{\circ} \mathrm{C}$.

FT-IR (KBr) v max.: 815, 1140, 1187, 1218, 2986, $3102 \mathrm{~cm}^{-1}$.

${ }^{1} \mathrm{H}$ NMR (400 MHz) (ס: ppm): $1.11\left(\mathrm{t}, 6 \mathrm{H},-\mathrm{CH}_{3}\right)$, $3.33\left(\mathrm{q}, 4 \mathrm{H},-\mathrm{CH}_{2}\right), 6.69(\mathrm{~d}, 2 \mathrm{H}, J=9.0 \mathrm{~Hz}, \mathrm{Ar}-\mathrm{H}), 6.71$ $(\mathrm{d}, 2 \mathrm{H}, J=9.1 \mathrm{~Hz}, \mathrm{Ar}-\mathrm{H}), 7.49(\mathrm{~d}, 2 \mathrm{H}, J=8.4 \mathrm{~Hz}, \mathrm{Ar}$ $-\mathrm{H}), 7.52(\mathrm{~d}, 2 \mathrm{H}, J=8.4 \mathrm{~Hz}, \mathrm{Ar}-\mathrm{H}), 8.03-8.16(\mathrm{~d}, 6 \mathrm{H}, J$ $=8.8,3.2 \mathrm{~Hz}, \mathrm{Ar}-\mathrm{H}), 8.39-8.40(\mathrm{~d}, 4 \mathrm{H}, J=8.8,2.5 \mathrm{~Hz}$, Ar $-\mathrm{H}), 8.43$ (s, 2H, Ethylenic protons).

${ }^{13}$ C NMR (100 MHz) (ס: ppm): 15.38, 47.94, 82.13, $115.32,118.81,119.06,121.17,124.0,129.23,133.4$, $139.28,145.43,149.30,152.34,155.13,164.40,173.9$, 177.3, 181.2.

Mass: $\mathrm{m} / \mathrm{e}=667.9(\mathrm{M}+1)$.

2E, 2'E-3,3'-((6-(4-(N,N-Diethylamino)phenyl)-1,3,5triazine-2,4-diyl)bis(oxy))bis(4,1-phenylene)bis(2-(4nitrophenyl)acrylonitrile) $7 d \quad 4,4$ '-((6-) (4- $(N, N-$ 
Diethylamino) phenyl)-1,3,5-triazine-2,4 diyl) bis (oxy)) dibenzaldehyde 5 (1.0 g, $0.0023 \mathrm{~mol})$ and 4-nitro phenyl acetonitrile $(0.84 \mathrm{~g}, 0.0052 \mathrm{~mol})$ were dissolved in absolute ethanol $(10 \mathrm{~mL})$. Piperidine $(0.1 \mathrm{~mL})$ was added to it and the reaction mixture was stirred at room temperature for $8 \mathrm{~h}$, filtered the reaction mass to separate out product and recrystallized product from ethanol.

Yield: $\left(1.40\right.$ g, $83 \%$, m.p.: $201^{\circ} \mathrm{C}$.

FT-IR (KBr) v max.: 513, 734, 836, 1562, 1635, 2279 , 2309, 2729, 3069, $3390 \mathrm{~cm}^{-1}$.

${ }^{1}$ H NMR (400 MHz) (ס: ppm): $1.10\left(\mathrm{t}, 6 \mathrm{H},-\mathrm{CH}_{3}\right)$, $3.32\left(\mathrm{q}, 4 \mathrm{H},-\mathrm{CH}_{2}\right), 6.66(\mathrm{~d}, 2 \mathrm{H}, J=9.0 \mathrm{~Hz}, \mathrm{Ar}-\mathrm{H}), 6.69$ (d, $2 \mathrm{H}, 7.39, J=9.09 \mathrm{~Hz}, \mathrm{Ar}-\mathrm{H}), 7.47(\mathrm{~d}, 2 \mathrm{H}, J=8.5$ $\mathrm{Hz}, \mathrm{Ar}-\mathrm{H}), 7.50(\mathrm{~d}, 2 \mathrm{H}, J=8.5 \mathrm{~Hz}, \mathrm{Ar}-\mathrm{H}), 8.03-8.12$ (d, $8 \mathrm{H}, J=8.8,3.2 \mathrm{~Hz}, \mathrm{Ar}-\mathrm{H}), 8.35-8.36(\mathrm{~d}, 4 \mathrm{H}, J=8.8$, $2.5 \mathrm{~Hz}, \mathrm{Ar}-\mathrm{H}$ ), 8.39 (s, 2H, Ethylenic protons).

${ }^{13}$ C NMR (100 MHz) (ס: ppm): 12.38, 43.94, 81.13, $110.72,113.28,114.26,119.17,122.87,128.91,131.4$, $132.28,143.67,148.30,151.64,155.84,160.40,171.39$, 174.6, 177.5 .

Mass: $\mathrm{m} / \mathrm{e}=757.3(\mathrm{M}+1), 758.3(\mathrm{M}+2)$.

2E, 2'E-3,3'-((6-(4-(N,N-Diethylamino)phenyl)-1,3,5triazine-2,4-diyl)bis(oxy))bis(4,1-phenylene)bis(2(benzo[d]thiazol-2-yl)acrylonitrile) 7e 4,4'-((6-(4-(N,NDiethylamino) phenyl)-1,3,5-triazine-2,4 diyl) bis (oxy)) dibenzaldehyde 5 (1.0 g, $0.0023 \mathrm{~mol})$ and 2-cyano methyl benzothiazole $(0.90 \mathrm{~g}, 0.0052 \mathrm{~mol})$ were dissolved in absolute ethanol $(10 \mathrm{~mL})$. Piperidine $(0.1 \mathrm{~mL})$ was added to it and the reaction mixture was stirred at room temperature for $5 \mathrm{~h}$, filtered the reaction mass to separate out product and recrystallized product from ethanol.

Yield: (1.16 g, 67\%), m.p.: $196^{\circ} \mathrm{C}$.

FT-IR (KBr) v max.: 513, 727, 821, 1565, 1600, 2278, 2328, 2729, 3069, $3370 \mathrm{~cm}^{-1}$.

${ }^{1}$ H NMR (400 MHz) (ס: ppm): $1.12\left(\mathrm{t}, 6 \mathrm{H},-\mathrm{CH}_{3}\right)$, $3.32\left(\mathrm{q}, 4 \mathrm{H},-\mathrm{CH}_{2}\right), 6.67(\mathrm{~d}, 2 \mathrm{H}, J=9.09 \mathrm{~Hz}, \mathrm{Ar}-\mathrm{H})$, $6.70(\mathrm{~d}, 2 \mathrm{H}, 7.39, J=9.09 \mathrm{~Hz}, \mathrm{Ar}-\mathrm{H}), 7.50(\mathrm{~d}, 2 \mathrm{H}, J=$ $7.5 \mathrm{~Hz}, \mathrm{Ar}-\mathrm{H}), 7.52(\mathrm{~d}, 2 \mathrm{H}, J=7.5 \mathrm{~Hz}, \mathrm{Ar}-\mathrm{H}), 7.53-$ $7.57(\mathrm{~d}, 8 \mathrm{H}, J=8.2 \mathrm{~Hz}, \mathrm{Ar}-\mathrm{H}), 8.03-8.22(\mathrm{~d}, 4 \mathrm{H}, J=$ 9.09, 7.6 Hz, Ar -H), 8.46 (s, 2H, Ethylenic protons).

${ }^{13} \mathrm{C}$ NMR (100 MHz) (ס: ppm): 12.38, 43.94, 81.13, $110.72,113.28,114.26,119.17,122.90,128.99,130.92$, $132.38,147.98,149.31,151.64,155.84,158.32,160.41$, 171.28, 174.89 .

Mass: $\mathrm{m} / \mathrm{e}=781.9(\mathrm{M}+1), 782.9(\mathrm{M}+2)$.

2,2' - (2E, 2E')-((6-(4-( $N, N$-Diethylamino) phenyl)1,3,5-triazine-2,4-diyl)bis(oxy)) bis(4,1-phenylene)) bis(1-phenyl prop-2-en-3-yl-1-ylidene))dimalononitrile $7 \mathbf{f}$

4,4'-((6-(4-(N,N-Diethylamino) phenyl)-1,3,5-triazine2,4 diyl) bis (oxy)) dibenzaldehyde $5(1.0 \mathrm{~g}, 0.0023 \mathrm{~mol})$ and 2-(2-phenylprop-1-en-1-ylidene) malononitrile (0.87 g, $0.0052 \mathrm{~mol})$ were dissolved in absolute ethanol (10 $\mathrm{mL})$. Piperidine $(0.1 \mathrm{~mL})$ was added to it and the reaction mixture was stirred at room temperature for $5 \mathrm{~h}$, filtered the reaction mass to separate out product and recrystallized product from ethanol.

Yield: $(1.38$ g, $74 \%)$, m.p.: $146^{\circ} \mathrm{C}$.

FT-IR (KBr) v max.: 739, 846, 1635, 2309, 3070, 3396 $\mathrm{cm}^{-1}$.

${ }^{1} \mathrm{H}$ NMR (400 MHz) (ס: ppm): $1.12\left(\mathrm{t}, 6 \mathrm{H},-\mathrm{CH}_{3}\right)$, 3.35 (q, 4H, $\left.-\mathrm{CH}_{2}\right), 6.69(\mathrm{~d}, 2 \mathrm{H}, J=9.2 \mathrm{~Hz}, \mathrm{Ar}-\mathrm{H}), 6.72$ $(\mathrm{d}, 2 \mathrm{H}, J=9.2 \mathrm{~Hz}, \mathrm{Ar}-\mathrm{H}), 7.49$ (d, $2 \mathrm{H}, J=8.6 \mathrm{~Hz}, \mathrm{Ar}$ $-\mathrm{H}), 7.51(\mathrm{~d}, 2 \mathrm{H}, J=8.6 \mathrm{~Hz}, \mathrm{Ar}-\mathrm{H}), 8.05-8.14(\mathrm{~d}, 10 \mathrm{H}$, $J=8.8,3.2 \mathrm{~Hz}, \mathrm{Ar}-\mathrm{H}), 8.37-8.39(\mathrm{~d}, 6 \mathrm{H}, J=8.8,2.5 \mathrm{~Hz}$, Ar $-\mathrm{H}), 8.42$ (s, 2H, Ethylenic protons).

${ }^{13}$ C NMR (100 MHz) (ס: ppm): 14.12, 45.43, 85.23, 112.72, 114.32, 115.26, 122.17, 124.37, 128.91, 131.4, $135.83,147.73,150.30,151.64,156.42,165.41,173.3$, $174.16,176.15,179.20$

Mass: $\mathrm{m} / \mathrm{e}=769.9(\mathrm{M}+1)$.

\section{Conclusion}

In summary, we have developed an efficient and simple protocol for the synthesis of fluorescent chromophores containing triazine core moiety and electron withdrawing cyano/carboethoxy groups. The synthesized compounds were confirmed by FT-IR, ${ }^{1} \mathrm{H}$ NMR, ${ }^{13} \mathrm{C}$ NMR and Mass spectral analysis. From the emissive properties, it was concluded that the electronic coupling between donor and acceptor was sufficient to allow charge transfer in their molecules. Emission properties of synthesized compounds are sensitive towards the solvent polarity; it shows positive solvatochromism with increase in solvent polarity.

\section{Acknowledgements}

Authors are thankful to Indian Institute of Technology, Mumbai for NMR and Mass spectral analysis.

\section{Authors' contributions}

VSP contributed for synthesis, spectral analysis of all compounds under NS; VSP and VSP contributed for photophysical study under the guidance NS. All the authors read and approved the final manuscript.

\section{Competing interests}

The authors declare that they have no competing interests.

Received: 18 October 2011 Accepted: 6 December 2011 Published: 6 December 2011

\section{References}

1. Fox M: Introduction electron transfer: a critical link between sub disciplines in chemistry. Chem. Rev 1992, 92:365-368.

2. Lakowicz J: Probe design and chemical sensing. In Topics in fluorescence spectroscopy. Volume 4. Edited by: Lakowicz JR. Plenum Press, New York; 1994:501-504.

3. Balaganesan B, Wen S, Chen C: Synthetic study of tetramethyl julolidine-A key intermediate toward the synthesis of the red dopant DCJTB for OLED applications. Tetrahedron Lett 2003, 44:145-147. 
4. Yu G, Gao J, Hummelen J, Wudl F, Heeger A: Polymer photovoltaic cells: enhanced efficiencies via a network of internal donor-acceptor hetero junctions. Science 1995, 270:1789-1791.

5. Gold H: Fluorescent brightening agents. In The chemistry of synthetic dyes. Edited by: Venkataraman K. Academic press, New York; 1971:535-679.

6. Shirota Y: Organic materials for electronic and optoelectronic devices. $J$ Mater Chem 2000, 10:1-25.

7. Sonawane Y, Phadtare S, Borse B, Jagtap A, Shankarling G: Synthesis of diphenylamine-based novel fluorescent styryl compounds by knoevenagel condensation using a conventional method, biocatalyst and deep eutectic solvent. Org Lett 2010, 12:1256-1259.

8. Cowley D, Kane E, Todd R: Triazinylaniline derivatives as fluorescence probes. Part 1. absorption and fluorescence in organic solvents and in aqueous media in relation to twisted intramolecular charge-transfer state formation, hydrogen bonding and protic equilibria. J Chem Soc Perkin trans 1991, 2:1495-1500.

9. Gupta V, Padalkar V, Phtangare K, Patil V, Umape P, Sekar N: The synthesis and photo-physical properties of extended styryl fluorescent derivatives of $\mathrm{N}$-ethyl carbazole. Dyes and Pigment 2011, 88:378-384.

10. Albota M, Beljonne D, Bredas J, Ehrlich J, Heikal A, Hess S, Kogej T: Design of organic molecules with large two-photon absorption cross sections. Science 1998, 281:1653-1656.

11. Cheng Y, Lou J, Haul S, Kim T, Shi Z, Lao D, Tocker N, Tian Y, Dalton L, Reid J, Jen A: Large electro-optic activity and enhanced thermal stability from diarylaminophenyl-containing high- $\beta$ nonlinear optical chromophores. Chem Mater 2007, 19:1154-1165.

12. Sochova S, Akella A, Hesselink L: Synthesis and characterization of photochromic organic films for holographic recording. Optic Lett 1997, 22:919-921.

13. Fu H, Wu H, Hou X, Xiao F, Shao B: N-Aryl carbazole derivatives for nondoped red OLEDs. Synthetic Metals 2006, 156:809-814.

14. Zhu W, Meng X, Yang Y, Zhang Q, Xie Y, Tian H: Bisthienylethenes containing a benzothiadiazole unit as a bridge:photochromic performance dependence on substitution position. Chemistry: A European Journal 2010, 16:899-906.

15. Xia Z, Zhang Z, Su J, Zhang Q, Fung K, Lam M, Li K, Wong W, Cheah K Tian H, Chen C: Robust and highly efficient blue light-emitting hosts based on indene-substituted anthracene. J Mater Chem 2010, 20:3768-3774

16. Williams A, Winfield S, Miller J: Relative fluorescence quantum yields sing a computer controlled luminescence spectrometer. Analyst 1983, 108:1067-1070

doi:10.1186/1752-153X-5-77

Cite this article as: Padalkar et al: Synthesis and photo-physical properties of fluorescent 1,3,5-triazine styryl derivatives. Chemistry Central Journal 2011 5:77.

Publish with ChemistryCentral and every
scientist can read your work free of charge
"Open access provides opportunities to our
colleagues in other parts of the globe, by allowing
anyone to view the content free of charge."
W. Jeffery Hurst, The Hershey Company.
- available free of charge to the entire scientific community
- peer reviewed and published immediately upon acceptance
- cited in PubMed and archived on PubMed Central
- yours - you keep the copyright
Submit your manuscript here:
http://www.chemistrycentral.com/manuscript/

